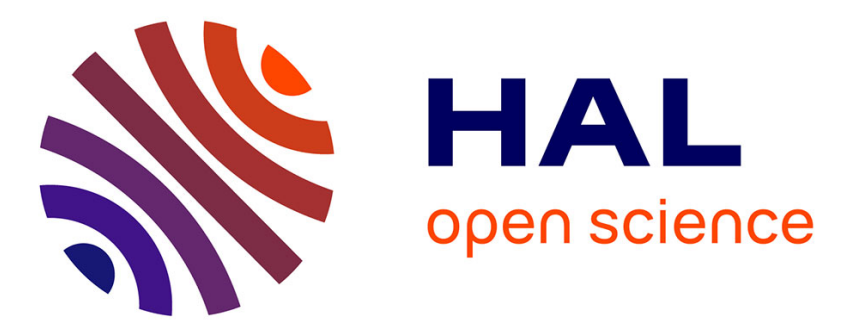

\title{
Performance Analysis of RIS-Aided Systems With Practical Phase Shift and Amplitude Response
}

\author{
Yan Zhang, Jiayi Zhang, Marco Di Renzo, Huahua Xiao, Bo Ai
}

\section{To cite this version:}

Yan Zhang, Jiayi Zhang, Marco Di Renzo, Huahua Xiao, Bo Ai. Performance Analysis of RISAided Systems With Practical Phase Shift and Amplitude Response. IEEE Transactions on Vehicular Technology, 2021, 70 (5), pp.4501 - 4511. 10.1109/tvt.2021.3069174 . hal-03377196

\section{HAL Id: hal-03377196 https://hal.science/hal-03377196}

Submitted on 14 Oct 2021

HAL is a multi-disciplinary open access archive for the deposit and dissemination of scientific research documents, whether they are published or not. The documents may come from teaching and research institutions in France or abroad, or from public or private research centers.
L'archive ouverte pluridisciplinaire HAL, est destinée au dépôt et à la diffusion de documents scientifiques de niveau recherche, publiés ou non, émanant des établissements d'enseignement et de recherche français ou étrangers, des laboratoires publics ou privés. 


\title{
Performance Analysis of RIS-Aided Systems With Practical Phase Shift and Amplitude Response
}

\author{
Yan Zhang (®) Jiayi Zhang (®), Senior Member, IEEE, Marco Di Renzo ${ }^{\circledR}$, Fellow, IEEE, Huahua Xiao, \\ and $\mathrm{Bo} \mathrm{Ai}^{\circledR}$, Senior Member, IEEE
}

\begin{abstract}
Reconfigurable intelligent surfaces (RISs) have drawn significant attention due to their capability of controlling the radio environment and improving the system performance. In this paper, we study the performance of an RIS-assisted single-input single-output system over Rayleigh fading channels. Differently from previous works that assume a constant reflection amplitude, we consider a model that accounts for the intertwinement between the amplitude and phase response, and derive closed-form expressions for the outage probability and ergodic capacity. Moreover, we obtain simplified expressions under the assumption of a large number of reflecting elements and provide tight upper and lower bounds for the ergodic capacity. Finally, the analytical results are verified by using Monte Carlo simulations.
\end{abstract}

Index Terms-Performance analysis, practical phase shift and amplitude response, reconfigurable intelligent surface.

\section{INTRODUCTION}

A RECONFIGURABLE intelligent surface (RIS) is an artificial planar structure with integrated electronic circuits, which is equipped with a large number of passive

Manuscript received December 17, 2020; revised February 23, 2021; accepted March 21, 2021. Date of publication March 26, 2021; date of current version June 9, 2021. This work was supported in part by the National Key R\&D Program of China under Grant 2020YFB1807201, in part by the National Natural Science Foundation of China under Grants 61971027, U1834210, and 61961130391, in part by Beijing Natural Science Foundation under Grant L202013, in part by Frontiers Science Center for Smart High-speed Railway System, in part by Royal Society Newton Advanced Fellowship under Grant NA191006, in part by the State Key Lab of Rail Traffic Control and Safety under Grants RCS2018ZZ007 and RCS2019ZZ007, in part by Open Research Fund of the State Key Laboratory of Integrated Services Networks under Grant ISN20-04, and in part by ZTE Corporation, and the State Key Laboratory of Mobile Network and Mobile Multimedia Technology. Marco Di Renzo's work was supported in part by European Commission through the H2020 ARIADNE project under Grant 871464 and through the H2020 RISE-6 G project under Grant 101017011. The review of this article was coordinated by Dr. C. Yuen. (Corresponding author: Jiayi Zhang.)

Yan Zhang and Jiayi Zhang are with the School of Electronic and Information Engineering, Beijing Jiaotong University, Beijing 100044, China and also with the Frontiers Science Center for Smart High-speed Railway System, Beijing 100044, China (e-mail: 20120168@bjtu.edu.cn; jiayizhang@bjtu.edu.cn).

Marco Di Renzo is with Université Paris-Saclay,CNRS, CentraleSupélec, Laboratoire des Signaux et Systèmes, 3 Rue Joliot-Curie, 91192 Gif-sur-Yvette, France (e-mail: marco.di-renzo@universite-paris-saclay.fr).

Huahua Xiao is with the ZTE Corporation, and State Key Laboratory of Mobile Network and Mobile Multimedia Technology, Shenzhen 518057, China (e-mail: xiao.huahua@zte.com.cn).

Bo Ai is with the State Key Laboratory of Rail Traffic Control and Safety, Beijing Jiaotong University, Beijing 100044, China and also with the Henan Joint International Research Laboratory of Intelligent Networking and Data Analysis, Zhengzhou University, Zhengzhou 450001, China (e-mail: boai@bjtu.edu.cn). and low-cost scattering elements that can effectively control the wireless propagation environment [1]. By intelligently adapting the phase shifts and the amplitude response of the scattering elements of an RIS, the signals reflected from it can be added constructively or destructively with other signals so as to enhance the signal strength or to suppress the co-channel interference at the receiver [2]-[12]. Thanks to these properties, RISs are considered to be a promising candidate technology for future wireless communication systems.

Several works have investigated the performance of RISassisted wireless systems [13]-[30]. In [13], the authors studied the coverage, the delay outage rate, and the probability of the signal-to-noise-ratio (SNR) gain of an RIS-assisted communication system over a Rayleigh fading channel by using the central limit theorem (CLT). In [14], exact and accurate approximated expressions for the bit error rate (BER) were derived over a Nakagami- $m$ fading channel. In [15] and [16], the authors studied the ergodic capacity (EC) of an RIS-assisted communication system. In [15], in particular, the impact of phase errors was analyzed. In [17], the outage probability (OP) was computed and minimized by optimizing the phase shifts of an RIS over Rician fading channels. In [18], an RIS was considered for assisting the communication between two users, and the OP and spectral efficiency were studied by using a Gamma approximation over Rayleigh fading channels. In [19], exact expressions of the OP and EC for an RIS-assisted system over Fox's $H$ fading channels were provided. In [20], the authors analyzed the impact of phase noise on the BER over Rayleigh fading channels. In [21], the authors quantified the impact of discrete phase shifts on the achievable rate over Rician fading channels. In [22], the authors analyzed the impact of phase noise and hardware impairments for transmission over line-of-sight (LoS) channels. In [23], the authors studied the impact of discrete phase shifts for achieving the full diversity order over Rayleigh fading channels. In [24], the authors investigated the ergodic secrecy capacity in the presence of discrete phase shifts and phase noise. In [25], the authors studied the secrecy outage probability of an RIS-assisted communication system over Rayleigh fading channels. In [26], the authors introduced a tight approximation for the distribution of the SNR in RIS-assisted communications that is formulated in terms of the squared $K_{G}$ distribution. In [27], the authors proposed a general framework to calculate the distribution of the SNR in multiple-antenna RIS-assisted systems in the presence of phase noise. In [28], the authors maximized the energy efficiency of an RIS-assisted downlink 
multi-user system by jointly designing the power allocation and phase shifts. In [29], the authors proposed a hybrid beamforming scheme for multi-hop RIS-assisted communication systems in order to improve the coverage range in the terahertz frequency band. In [30], the authors analyzed the impact of phase noise on the OP of RIS-assisted communication systems over generalized fading channels.

Although there exist several contributions that analyze the performance of RIS-assisted systems, most of them are based on approximations. For instance, the CLT approximation is frequently used but it is only accurate when the number of reflecting elements of the RIS is sufficiently large. Additionally, to the best of the authors' knowledge, no analytical studies have been conducted to investigate the system performance of RIS-assisted transmission by taking into account the interplay between the amplitude and phase response of each reflecting element of the RIS [31]. The above mentioned works, in particular, assume that the amplitude response of each reflecting element is independent of the applied phase shift, which is not always possible as discussed in [31] and [32]. In [32], in particular, it is shown that the amplitude and phase responses are, in general, intertwined and depend on the circuital model of the tuning circuit that controls each reflecting element of the RIS.

Motivated by these considerations, in this paper, we present a detailed performance analysis of RIS-assisted single-input single-output (SISO) systems over Rayleigh fading channels, by taking into account the intertwinement between the amplitude and phase response of the reflecting elements of the RIS. In particular, exact closed-form expressions for the OP and EC are derived by considering the amplitude and phase model empirically derived in [31]. In addition, in order to gain design insights, simplified expressions are obtained in some asymptotic regimes. Furthermore, we characterize the impact of key parameters on the system performance. The obtained findings show that an increase of the transmit SNR, the number of elements of the RIS as well as the minimum reflecting amplitude help improve the performance. It is shown, on the other hand, that the performance degrades with an increase of the steepness of the amplitude response. The main contributions of this paper can be summarized as follows.

- We provide a new analytical framework for the performance analysis of RIS-assisted systems. Considering a practical model for the phase shift and amplitude response, we derive exact closed-form expressions for the OP and the EC.

- In order to get additional insights into the impact of system parameters, we present asymptotic expressions for the $\mathrm{OP}$ and EC under the assumption of a large number of reflecting elements. In addition, we derive upper and lower bounds for the EC. The accuracy of the bounds becomes tighter when the number of reflecting elements of the RIS grows large.

- Capitalizing on the achieved analytical results, we analyze the impact of the transmit SNR, the number of elements of the RIS, the minimum reflecting amplitude as well as the steepness of the amplitude response on the RIS-assisted system performance.

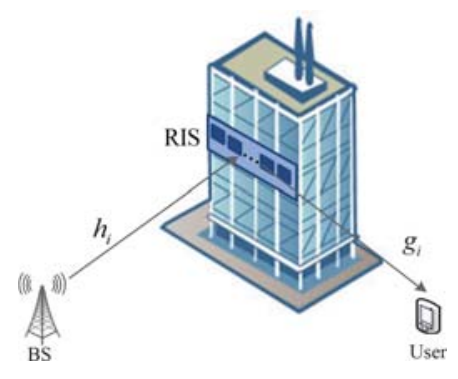

Fig. 1. RIS-assisted communication system.

The remainder of this paper is organized as follows. In Section II, we introduce the system and channel models. In Section III, exact closed-form expressions for the OP and the EC are obtained. Also, asymptotic expressions when the number of reflecting elements of the RIS is sufficiently large are obtained. Moreover, tight upper and lower bounds for the EC are derived. In Section IV, some numerical and simulation results are presented to confirm the accuracy of the derived expressions. Finally, Section V concludes the paper and reports some future research directions.

\section{SYSTEM MODEL}

As illustrated in Fig. 1, we consider a $\mathrm{SISO}^{1}$ system in which a single-antenna base station (BS) serves a single-antenna user. The communication is assisted by an RIS which is installed on the facade of a building. The RIS comprises $N$ nearly-passive reflecting elements and one micro-controller. The $N$ reflecting elements are assumed to be spaced half of the wavelength apart. The signals transmitted from the BS are appropriately reflected by the RIS by dynamically adjusting the phase shifts of the reflecting elements. We assume that the direct link from the BS to the user is blocked due to the presence of obstacles, such as buildings. In addition, we make the assumption that full channel state information (CSI) is available at the BS in order to optimize the phase shifts. Also, we consider only the signal reflected by the RIS the first time and ignore the signals reflected by the RIS two or more times.

We denote by $h_{i} \triangleq \alpha_{i} e^{-j \varphi_{i}}(i=1,2, \ldots, N)$ the baseband equivalent fading channel between the BS and the $i$-th element of the RIS, and by $g_{i} \triangleq \beta_{i} e^{-j \psi_{i}}$ the fading channel between the $i$-th element of the RIS and the user. Furthermore, $h_{i}$ and $g_{i}$ are assumed to be independent and identically distributed (i.i.d.) complex Gaussian random variables (RVs) with zero mean and $\sigma_{0}^{2}=1 / 2$ variance. Therefore, the magnitudes of $h_{i}$ and $g_{i}$ (i.e., $\alpha_{i}$ and $\beta_{i}$ ) follow the Rayleigh distribution and the phases $\varphi_{i}$ and $\psi_{i}$ are uniformly distributed in $[-\pi, \pi)$. Hence, the received signal $y$ at the user can be expressed as

$$
y=\sqrt{P_{s}}\left(\sum_{i=1}^{N} h_{i} v_{i} g_{i}\right) x+n,
$$

where $P_{s}$ is the transmit power at the BS, $x$ is the transmit signal with unit energy, and $n$ is the zero-mean additive white Gaussian noise (AWGN) whose variance is $N_{0}$. Additionally,

\footnotetext{
${ }^{1}$ The generalization of this work to multi-antenna transceivers is a promising direction for future research.
} 
$v_{i} \triangleq \rho_{i}\left(\phi_{i}\right) e^{j \phi_{i}}$ is the reflection coefficient applied by the $i$-th reconfigurable element of the RIS, where $\phi_{i} \in[-\pi, \pi)$ denotes the induced phase shift and $\rho_{i}\left(\phi_{i}\right) \in[0,1]$ denotes the induced amplitude which is dependent on the phase shift. In general, the relation between the phase and amplitude applied by the reconfigurable elements of the RIS depends on the load or surface impedances as elaborated in [4] and [32]. In this paper, we consider the closed-form empirical model that was developed in [31]. According to [31], in particular, the amplitude response $\rho_{i}\left(\phi_{i}\right)$ can be explicitly expressed, as a function of the phase shift, as

$$
\rho_{i}\left(\phi_{i}\right) \triangleq\left(1-\kappa_{\min }\right)\left(\frac{\sin \left(\phi_{i}-\vartheta\right)+1}{2}\right)^{\xi}+\kappa_{\text {min }},
$$

where $\kappa_{\min } \geq 0$ is the minimum amplitude, $\vartheta \geq 0$ is the horizontal distance between $-\pi / 2$ and $\kappa_{\min }$, which corresponds to the difference between $-\pi / 2$ and the phase shift $\phi_{i}$ that minimizes $\sin \left(\phi_{i}-\vartheta\right)$ (i.e., when $\phi_{i}=-\pi / 2+\vartheta$ ), and $\xi$ is the steepness of the function curve. It is worth noting that (2) yields an ideal phase shift model (i.e., $\rho_{i}\left(\phi_{i}\right)=1$ ) if $\kappa_{\min }=1$ or $\xi=0$. From (1) and (2), the instantaneous end-to-end SNR at the user can be expressed as

$$
\gamma=\left|\sum_{i=1}^{N} \alpha_{i} \beta_{i} \rho_{i}\left(\phi_{i}\right) e^{j\left(\phi_{i}-\varphi_{i}-\psi_{i}\right)}\right|^{2} \gamma_{t},
$$

where $|\cdot|$ denotes the absolute value, $\gamma_{t} \triangleq P_{s} / N_{0}$ represents the SNR. By setting $\phi_{i}=\varphi_{i}+\psi_{i}$ for $i=1, \ldots, N$ to fully compensate the channel phases ${ }^{2}$, (3) simplifies to

$$
\gamma=A^{2} \gamma_{t}
$$

where $A \triangleq \sum_{i=1}^{N} \alpha_{i} \beta_{i} \rho_{i}\left(\varphi_{i}+\psi_{i}\right)$.

It is worth mentioning that the SNR in (4) is not necessarily the maximum achievable SNR, since $\rho(\cdot)$ depends on the phase shift [31]. The SNR in (4) provides information on the actual SNR that is obtained by ignoring the interplay between the phase and amplitude response of the reflecting elements of the RIS at the optimization stage. The objective of the present paper is, in

\footnotetext{
${ }^{2}$ As for an RIS-assisted communication system in the presence of direct link, the phase shifts at the RIS can be designed in a way that the reflected signals from the RIS and the direct signal are co-phased [25]. More specifically, the approach proposed in this paper can be applied to analyze the performance of RIS-assisted communication systems in the presence of the direct link. This is left to a future research work.
}

fact, to assess the performance of RIS-assisted systems under this considered mismatched design and to quantify the impact of ignoring the interplay between the amplitude and the phase response.

\section{Performance Analysis}

\section{A. Outage Probability}

Define $Z_{i} \triangleq \alpha_{i} \beta_{i}$, the probability density function (PDF) of $Z_{i}$ is given by [33, Eq. (3)]

$$
f_{Z_{i}}(z)=4 z K_{0}(2 z)
$$

where $K_{\nu}($.$) is the modified \nu$-order Bessel function of the second kind [34, Eq. (8.432)]. By utilizing [35, Eq. (07.34.03.0605.01)], we can rewrite (5) in terms of the Meijer's $G$-function [34, Eq. (9.301)] as

$$
f_{Z_{i}}(z)=2 G_{0,2}^{2,0}\left[\begin{array}{l|l}
z^{2} & - \\
\frac{1}{2}, \frac{1}{2}
\end{array}\right] .
$$

Define $R_{i} \triangleq \rho_{i}\left(\varphi_{i}+\psi_{i}\right)=\left(1-\kappa_{\min }\right)\left(\frac{\sin \left(\varphi_{i}+\psi_{i}-\vartheta\right)+1}{2}\right)^{\xi}+$ $\kappa_{\text {min }}$. It is not difficult to show that $\varphi_{i}+\psi_{i}$ is uniformly distributed in $[-\pi, \pi)$. Since $R_{i}$ is a function of $\varphi_{i}+\psi_{i}$, the PDF of $R_{i}$ for $\kappa_{\min } \neq 1$ and $\xi \neq 0$ can be formulated by using the transformation method between two RVs [36, Eq. (2.1.49)] as

$$
f_{R_{i}}(r)=\frac{\left(\frac{r-\kappa_{\min }}{1-\kappa_{\min }}\right)^{1 / \xi-1}}{\pi \xi\left(1-\kappa_{\min }\right) \sqrt{\left(\frac{r-\kappa_{\min }}{1-\kappa_{\min }}\right)^{1 / \xi}-\left(\frac{r-\kappa_{\min }}{1-\kappa_{\min }}\right)^{2 / \xi}}},
$$

where $r \in\left(\kappa_{\min }, 1\right)$. If $\kappa_{\min }=1$ and/or $\xi=0$, then $R_{i}=1$ and the PDF of $R_{i}$ is independent of $\vartheta$.

Corollary 1: The cumulative distribution function (CDF) of the end-to-end SNR for an RIS-assisted system is given in (8) at the bottom of the page, where $H[\cdot, \ldots, \cdot]$ is the multivariable Fox's $H$-function [37, Eq. (A.1)]. In (8), in particular, we define $\tau \triangleq k \bmod 3$ and have

$$
\left\{\begin{array}{l}
\tau=1: m_{k}=0, n_{k}=2, p_{k}=2, q_{k}=0 ; \\
\text { and } c_{1}^{(k)}=0, \gamma_{1}^{(k)}=1, c_{2}^{(k)}=0, \gamma_{2}^{(k)}=1 ; z_{k}=\left(\frac{\sqrt{\gamma_{t}} \kappa_{\min }}{\sqrt{\gamma}}\right)^{2} \\
\tau=2: m_{k}=0, n_{k}=1, p_{k}=1, q_{k}=1 ; \\
\text { and } c_{1}^{(k)}=0, \gamma_{1}^{(k)}=\frac{1}{2} ; d_{1}^{(1)}=\frac{1}{2}, \delta_{1}^{(1)}=\frac{1}{2}, z_{k}=1 \\
\tau=0: m_{k}=0, n_{k}=1, p_{k}=1, q_{k}=0 ; \\
\text { and } c_{1}^{(k)}=0, \gamma_{1}^{(k)}=\frac{1}{2 \xi} ; z_{k}=a^{-\frac{1}{2 \xi}}
\end{array}\right.
$$

$$
\begin{aligned}
& F_{\gamma}(\gamma)=\left(\frac{1}{\sqrt{\pi} \xi}\right)^{N} H_{2 N, N+1: p_{1}, q_{1}: \ldots: p_{k}, q_{k}: \ldots: p_{3 N}, q_{3 N}}^{0,2 N: m_{1}, n_{1}: \ldots: m_{k}, n_{k}: m_{3 N}, n_{3}} \\
& \times\left[\begin{array}{l}
\left(a_{j} ; \alpha_{j}^{(1)}, \ldots, \alpha_{j}^{(k)}, \ldots, \alpha_{j}^{(3 N)}\right)_{1,2 N}:\left(c_{j}^{(1)}, \gamma_{j}^{(1)}\right)_{1, p_{1}} ; \ldots ;\left(c_{j}^{(3 N)}, \gamma_{j}^{(3 N)}\right)_{1, p_{3 N}} \\
(0 ;-2,0,0, \ldots,-2,0,0)\left(b_{j} ; \beta_{j}^{(1)}, \ldots, \beta_{j}^{(k)}, \ldots, \beta_{j}^{(3 N)}\right)_{1, N}:\left(d_{j}^{(1)}, \delta_{j}^{(1)}\right)_{1, q_{1}} ; \ldots ;\left(d_{j}^{(3 N)}, \gamma_{j}^{(3 N)}\right)_{1, q_{3 N+1}}
\end{array} \mid\right. \\
& \left.z_{1}, \ldots, z_{k}, \ldots, z_{3 N}\right]
\end{aligned}
$$


In particular, if $j$ is even, i.e., $j$ can be expressed as $j=$ $2 \omega(\omega=1,2, \ldots, N)$, we have $a_{j}=0, \alpha_{j}^{(3 \omega-1)}=-1, \alpha_{j}^{(3 \omega)}=$ -1 , and $\alpha_{j}^{(k)}=0$ for $k \neq 3 \omega-1,3 \omega$. Otherwise, if $j$ is odd, i.e., $j=2 \omega-1$, we have $a_{j}=1, \alpha_{j}^{(3 \omega-2)}=-2, \alpha_{j}^{(3 \omega)}=-\frac{1}{2 \xi}$, for $k \neq 3 \omega-2,3 \omega, \alpha_{j}^{(k)}=0$. Furthermore, if $k \bmod 3=1$, we have $b=1$ and $\beta^{(k)}=-2$, for other $k, \beta^{(k)}=0$. Also, $b_{j}=-1$, $\beta_{j}^{(3 \omega-1)}=-1, \beta_{j}^{(3 \omega)}=-1$ and $\beta_{j}^{(3 \omega-2)}=0$.

Proof: See Appendix A.

The multivariate Fox's $H$-function in (8) can be calculated by using widely used mathematical software tools, such as Mathematica [38], [39] and Matlab [40, Appendix C]. The Python implementation of this function is provided in [41].

The OP is defined as the probability that the instantaneous end-to-end SNR $\gamma$ falls below a given threshold $\gamma_{\text {th }}$. From (8), shown at the bottom of the next page, the OP can be directly obtained by setting $\gamma=\gamma_{\mathrm{th}}$, as follows

$$
P_{\text {out }}=\operatorname{Pr}\left(\sum_{i=1}^{N} Z_{i} R_{i} \leq \sqrt{\frac{\gamma_{\mathrm{th}}}{\gamma_{t}}}\right)=F_{\gamma}\left(\gamma_{\mathrm{th}}\right) .
$$

1) Large $N$ Approximation: For a sufficiently large number of reflecting elements, i.e., $N \gg 1$, according to the CLT, $A$ converges to a Gaussian distributed RV. In this case, a simplified expression of the OP can be obtained by computing the mean and variance of $A$. To this end, we note that the mean and variance of $Z_{i}$ are $\mathbb{E}\left(Z_{i}\right)=\pi / 4$ and $\operatorname{Var}\left(Z_{i}\right)=\left(16-\pi^{2}\right) / 16$ [6], respectively. Accordingly, we provide the following corollary.

Corollary 2: The mean value $\varepsilon$ and the variance $\delta$ of $R_{i}$ are given by

$$
\begin{aligned}
& \varepsilon \triangleq \mathbb{E}\left(R_{i}\right)=\frac{1-\kappa_{\min }}{\pi} B\left(\xi+\frac{1}{2}, \frac{1}{2}\right)+\kappa_{\text {min }}, \\
& \delta \triangleq \operatorname{Var}\left(R_{i}\right)=\frac{\left(1-\kappa_{\min }\right)^{2}}{\pi} B\left(2 \xi+\frac{1}{2}, \frac{1}{2}\right) \\
& -\left(\frac{1-\kappa_{\text {min }}}{\pi}\right)^{2} B^{2}\left(\xi+\frac{1}{2}, \frac{1}{2}\right),
\end{aligned}
$$

where $B(\cdot, \cdot)$ is the beta function defined in [34, Eq. (8.384)], $\mathbb{E}(\cdot)$ and $\operatorname{Var}(\cdot)$ represent the statistical expectation and the variance, respectively.

Proof: See Appendix B.

Since $Z_{i}$ and $R_{i}$ are independent of each other, the mean and variance of $A$ are, respectively, equal to

$$
\begin{aligned}
& \qquad \mathbb{E}(A)=N \pi \varepsilon / 4, \\
& \operatorname{Var}(A)=N\left(\delta+\varepsilon^{2}\left(1-\pi^{2} / 16\right)\right) \\
& \text { Therefore, } A^{2} \text { can be approximated with a non-central chi- } \\
& \text { square RV with one degree of freedom whose PDF is [13, Eq. } \\
& \text { (4)] } \\
& f_{A^{2}}(x)=\frac{1}{2 \eta^{2}}\left(\frac{x}{\lambda}\right)^{-\frac{1}{4}} \exp \left(-\frac{x+\lambda}{2 \eta^{2}}\right) I_{-\frac{1}{2}}\left(\frac{\sqrt{x \lambda}}{\eta^{2}}\right),
\end{aligned}
$$
(4)] where $I_{\nu}($.$) is the modified Bessel function of the first kind [34,$ Eq. (8.406)], $\lambda \triangleq\left(\frac{N \pi \varepsilon}{4}\right)^{2}$, and $\eta^{2} \triangleq N\left(\delta+\varepsilon^{2}\left(1-\frac{\pi^{2}}{16}\right)\right)$. From (4), therefore, the PDF of $\gamma$ can be formulated as

$$
f_{\gamma}(\gamma)=\frac{1}{2 \gamma_{t} \eta^{2}}\left(\frac{\gamma}{\gamma_{t} \lambda}\right)^{-\frac{1}{4}} \exp \left(-\frac{\gamma+\lambda \gamma_{t}}{2 \gamma_{t} \eta^{2}}\right) I_{-\frac{1}{2}}\left(\frac{\sqrt{\gamma \lambda}}{\sqrt{\gamma_{t}} \eta^{2}}\right)
$$

Finally, the OP can be obtained by using [42, Eq. (3)], $F_{\gamma}(\gamma)=$ $\int_{0}^{\gamma} f_{\gamma}(\gamma) d \gamma$ and (9), as follows

$$
P_{\text {out }}=1-Q_{\frac{1}{2}}\left(\frac{\sqrt{\lambda}}{\eta}, \frac{\sqrt{\gamma_{\mathrm{th}}}}{\sqrt{\overline{\gamma_{t}}} \eta}\right)
$$

where $Q_{m}(\cdot, \cdot)$ is the Marcum $Q$-function [42, Eq. (3)]. Equation (14) provides a more efficient approach than (9) to calculate the OP when $N \rightarrow \infty$. Although (14) is obtained under the assumption of large $N$, it is sufficiently tight even for moderate values of $N$ in the low-SNR regime, as it is shown in the numerical results.

\section{B. Ergodic Capacity}

In this subsection, we analyze the EC defined as

$$
C=\mathbb{E}\left[\log _{2}(1+\gamma)\right]=\int_{0}^{\infty} \log _{2}(1+\gamma) f_{\gamma}(\gamma) d \gamma
$$

Corollary 3: The ergodic capacity in (15) is given in (16), shown at the bottom of the next page, where for $j=1, \ldots, 2 N$, the parameters $a_{j}, \alpha_{j}^{(k)}$ are the same as in (8), while for other values of $j$, we have

$$
\left\{\begin{array}{r}
a_{2 N+1}=1, \alpha_{2 N+1}^{(3 \omega-2)}=-2, \alpha_{2 N+1}^{(3 N+1)}=1 \\
\text { for } k \neq 3 \omega-2, \alpha_{2 N+1}^{(k)}=0 \\
a_{2 N+2}=0, \alpha_{2 N+2}^{(3 \omega-2)}=1, \alpha_{2 N+2}^{(3 N+1)}=-1 \\
\text { for } k \neq 3 \omega-2, \alpha_{2 N+2}^{(k)}=0 \\
a_{2 N+3}=0, \alpha_{2 N+3}^{(3 \omega-2)}=1, \alpha_{2 N+3}^{(3 N+1)}=-1 \\
\text { for } k \neq 3 \omega-2, \alpha_{2 N+3}^{(k)}=0
\end{array}\right.
$$

Furthermore, for $j=1, \ldots, N, b_{j}$ and $\beta_{j}^{(k)}$ are the same as in (8), for $j=N+1$, we have $b_{N+1}=-1, \beta_{N+1}^{(3 \omega-2)}=$ $1, \beta_{N+1}^{(3 N+1)}=-1$, and for $k \neq 3 \omega-2$, we have $\beta_{N+1}^{(k)}=$ 0 . In addition, $c_{j}^{(k)}, \gamma_{j}^{(k)}, d_{j}^{(k)}$ and $\delta_{j}^{(k)}$ are the same as in (8) but $z_{k}=\left(\sqrt{\gamma_{t}} \kappa_{\min }\right)^{2}$ when $k \bmod 3=1$. Also, for $k=3 N+1$, we have $m_{3 N+1}=0, n_{3 N+1}=1, p_{3 N+1}=$ $1, q_{3 N+1}=0 ; c_{1}^{(3 N+1)}=0, \gamma_{1}^{(3 N+1)}=-1, z_{k}=s$.

Proof: See Appendix C.

1) Bounds for the Ergodic Capacity: The exact expression of the ergodic capacity in Corollary 3 is not simple enough to gain engineering insights. Therefore, we introduce upper and lower bounds according to Jensen's inequality as follows

$C^{\mathrm{lb}} \triangleq \log _{2}\left(1+(\mathbb{E}(1 / \gamma))^{-1}\right) \leq C \leq C^{\mathrm{ub}} \triangleq \log _{2}(1+\mathbb{E}(\gamma))$ 
Corollary 4: The ergodic capacity of the considered RISassisted communication system is upper bounded by

$$
\mathbb{E}(\gamma)=\gamma_{t} N\left(\delta+\varepsilon^{2}\right)+\gamma_{t} \frac{N(N-1) \pi^{2} \varepsilon^{2}}{16} .
$$

The lower bound for the ergodic capacity can be approximated as

$$
C^{\mathrm{lb}} \approx \log _{2}\left\{1+\left(1 / E(\gamma)+\operatorname{Var}(\gamma) /[E(\gamma)]^{3}\right)^{(-1)}\right\},
$$

where

$$
\begin{aligned}
\operatorname{Var}(\gamma) & =N \gamma_{t}^{2}\left[4 \varpi-\left(\varepsilon^{2}+\delta\right)^{2}\right]+2 N(N-1) \gamma_{t}^{2} \\
& \times\left[\left(\delta+\varepsilon^{2}\right)^{2}-\frac{\pi^{4} \varepsilon^{4}}{256}\right] \\
& +N(N-1) \gamma_{t}^{2}\left(\frac{9 \pi^{2} \nu \varepsilon}{16}-\frac{\pi^{2} \varepsilon^{2}\left(\varepsilon^{2}+\delta\right)}{4}\right) \\
& +N(N-1)(N-2) \gamma_{t}^{2}\left(\frac{\pi^{2} \varepsilon^{2}\left(\varepsilon^{2}+\delta\right)}{4}-\frac{\pi^{4} \varepsilon^{4}}{64}\right), \\
& \varpi \frac{\triangleq\left(1-\kappa_{\min }\right)^{4}}{\pi} B\left(4 \xi+\frac{1}{2}, \frac{1}{2}\right) \\
& +\frac{4 \kappa_{\min }\left(1-\kappa_{\min }\right)^{3}}{\pi} B\left(3 \xi+\frac{1}{2}, \frac{1}{2}\right) \\
& +\frac{6 \kappa_{\min }^{2}\left(1-\kappa_{\min }\right)^{2}}{\pi} B\left(2 \xi+\frac{1}{2}, \frac{1}{2}\right) \\
& +\frac{4 \kappa_{\min }^{3}\left(1-\kappa_{\min }\right)}{\pi} B\left(\xi+\frac{1}{2}, \frac{1}{2}\right)+\kappa_{\min }^{4}, \\
& \triangleq \\
\qquad & \frac{3 \kappa_{\min }^{2}\left(1-\kappa_{\min }\right)}{\pi} B\left(\xi+\frac{1}{2}, \frac{1}{2}\right)+\kappa_{\min }^{3} \\
+ & \left.\left(1-\kappa_{\min }\right)^{3} B\left(3 \xi+\frac{1}{2}, \frac{1}{2}\right)+\frac{3\left(1-\kappa_{\min }\right)^{2} \kappa_{\min }}{\pi}, \frac{1}{2}\right) \\
& (20)
\end{aligned}
$$

\section{Proof: See Appendix D.}

When $\xi$ is fixed, we rewrite (10) as $\varepsilon=f\left(\kappa_{\min }\right) \triangleq$ $\left(1-\frac{\Gamma\left(\xi+\frac{1}{2}\right)}{\sqrt{\pi} \Gamma(\xi+1)}\right) \kappa_{\min }+\frac{\Gamma\left(\xi+\frac{1}{2}\right)}{\sqrt{\pi} \Gamma(\xi+1)}$ using [34, Eq. (8.384.1)]. Differentiating $f\left(\kappa_{\min }\right)$ with respect to $\kappa_{\min }$, we obtain $f^{\prime}\left(\kappa_{\min }\right)=1-\frac{\Gamma\left(\xi+\frac{1}{2}\right)}{\sqrt{\pi} \Gamma(\xi+1)}$. Then, let us define $f(\xi) \triangleq \frac{\Gamma\left(\xi+\frac{1}{2}\right)}{\sqrt{\pi} \Gamma(\xi+1)}$. Its derivative can be expressed as $f^{\prime}(\xi)=\frac{\Gamma\left(\xi+\frac{1}{2}\right)}{\sqrt{\pi} \Gamma(\xi+1)}\left(\psi\left(\xi+\frac{1}{2}\right)-\psi(\xi+1)\right)$, where $\psi($.$) is$ the psi function [34, Eq. (8.360.1)]. Employing [34, Eq. (8.363.3)], $\psi\left(\xi+\frac{1}{2}\right)-\psi(\xi+1)$ can be written as $\sum_{k=0}^{\infty}\left(\frac{1}{\xi+1+k}-\frac{1}{\xi+\frac{1}{2}+k}\right)$, from which we conclude that $f^{\prime}(\xi)<0$ always holds. As such, we obtain $f(\xi)<f(0)=1$ and $f^{\prime}\left(\kappa_{\min }\right)>0$, i.e., $\varepsilon$ increases monotonically for $\kappa_{\min } \in(0,1)$ when $\xi$ is a constant. Similarly, it can be proved that $\delta$ increases when $\kappa_{\min }$ increases and $\xi$ is fixed. In addition, when $\kappa_{\min }$ remains unchanged, both $\varepsilon$ and $\delta$ decreases as $\xi$ increases. As a result, it can be observed form (18) that the upper bound of the EC increases as $N$ increases. Furthermore, increasing $\kappa_{\min }$ or decreasing $\xi$ with the other parameters being fixed, which corresponds to an increase of the reflection amplitude, further increases the upper bound of the EC. Moreover, it can be proved that the lower bound of the EC is improved when $\kappa_{\min }$ increases or $\xi$ decreases.

2) Large $N$ Approximation:

Corollary 5: For a large number of the reflecting elements, the EC can be approximated as

$$
\begin{aligned}
& C=\frac{B \pi}{2 \sqrt{\eta} \ln 2}\left(\frac{\gamma^{3}}{8 \lambda \gamma_{t}^{3}}\right)^{-\frac{1}{4}} \exp \left(-\frac{\lambda}{2 \eta^{2}}\right) H_{1,0: 1,3: 2,2}^{0,1: 1,0: 1,2} \times \\
& {\left[\begin{array}{ll}
\left(\frac{1}{4} ; 1,1\right)\left|\left(\begin{array}{l}
\left.\frac{1}{4}, 1\right) \\
-
\end{array}-\frac{1}{4}, 1\right),\left(\frac{1}{4}, 1\right),\left(\frac{1}{4}, 1\right)\right| \begin{array}{l}
(1,1),(1,1) \\
(1,1),(0,1)
\end{array} \mid \frac{\lambda}{2 \eta^{2}}, 2 \gamma_{t} \eta^{2}
\end{array}\right] .}
\end{aligned}
$$

Proof: See Appendix E.

Although (21) is obtained under the assumption of large $N$, the asymptotic result provides a good tightness even for moderate values of $N$, as substantiated by the numerical results.

\section{NUMERICAL RESUlTS}

In this section, Monte Carlo simulations are presented to verify the analytical expressions obtained in the previous section. The outage threshold $\gamma_{\text {th }}$ in Fig. 2 and Fig. 3 is set equal to $10 \mathrm{~dB}$.

In Fig. 2, the OP of the considered RIS-assisted system is shown for different values of $N$ and $\kappa_{\min }$. It can be observed from this figure that the analytical results closely overlap with the simulated results, which proves the accuracy of the analysis. Moreover, as expected, the system performance can be improved

$$
\begin{aligned}
& C=\frac{1}{2 s \ln 2}\left(\frac{1}{\sqrt{\pi} \xi}\right)^{N} H_{2 N+3, N+2: p_{1}, q_{1}: \ldots: m_{k}, q_{k}: \ldots: p_{3 N+1}, q_{3 N+1}}^{0,2 N+3: m_{1}, n_{1}: \ldots: m_{k}, n_{k}: \ldots: m_{3 N+1}, n_{3 N+1}}
\end{aligned}
$$

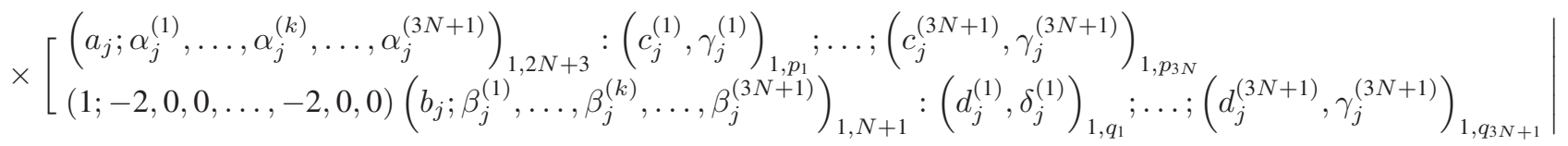

$$
\begin{aligned}
& \left.z_{1}, \ldots, z_{k}, \ldots, z_{3 N+1}\right]
\end{aligned}
$$




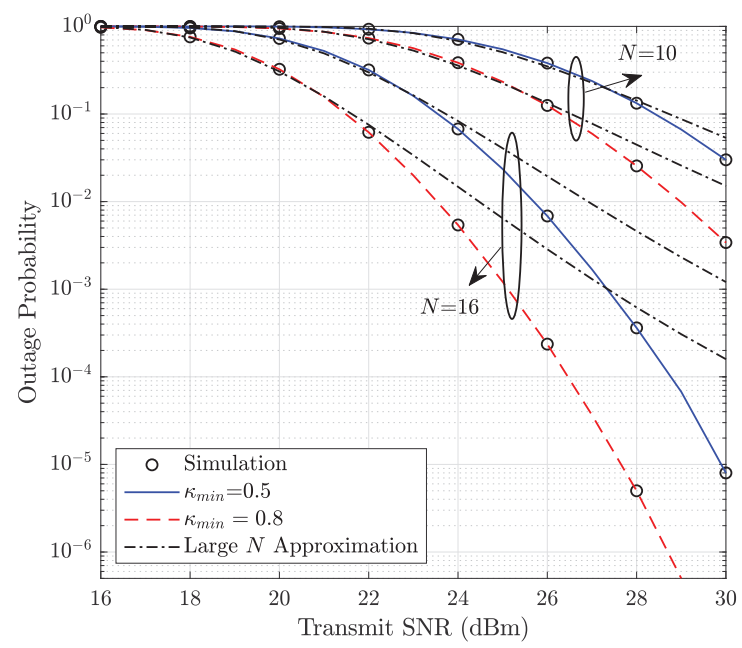

Fig. 2. Outage probability versus $\gamma_{t}$ for different $N$ and $\kappa_{\text {min }}(\xi=1.5)$.

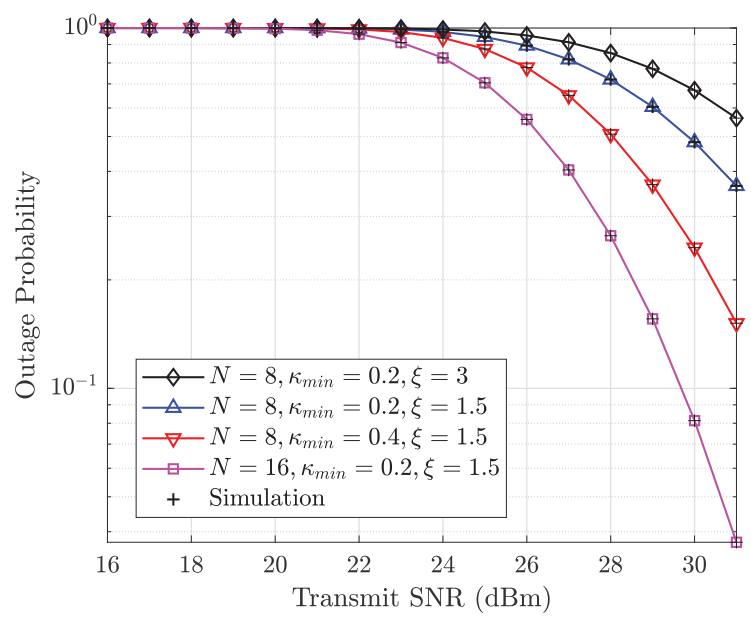

Fig. 3. Outage probability versus $\gamma_{t}$ for different $N, \kappa_{\min }$ and $\xi$.

by increasing $N$. Furthermore, the larger $\kappa_{\min }$ is, the smaller the OP. We can also observe that the system performance improves as the SNR increases. In addition, we observe that the CLT approximation is accurate even for moderate values of $N$ in the low SNR regime.

In Fig. 3, we analyze the $\mathrm{OP}$ as a function of $N, \kappa_{\min }$ and $\xi$. From Fig. 3, we observe that increasing $N$ and $\kappa_{\min }$ improves the OP. On the other hand, the OP degrades by increasing $\xi$. In the considered setup, based on Fig. 3, we can compare the impact of $N, \kappa_{\min }$ and $\xi$ on the OP. Take the transmit SNR of $30 \mathrm{dBm}$ for instance, we consider the $\mathrm{OP}$ when $N=8, \kappa_{\min }=0.2$ and $\xi=1.5$ as a reference. When we set $N=16$ with fixed values of $\kappa_{\min }$ and $\xi$, the OP decreases by $83 \%$. Similarly, the OP decreases by $49 \%$ when we double the value of $\kappa_{\text {min }}$ with fixed values of $N$ and $\xi$. Finally, the OP increases by $39 \%$ when the value of $\xi$ is doubled with fixed values of $N$ and $\kappa_{\text {min }}$. Therefore, we observe that $N$ has the greatest impact on the system performance, followed by $\kappa_{\min }$, and finally by $\xi$.

Fig. 4 illustrates the tightness of the upper and lower bounds of the EC. We observe that the performance gap between the upper and lower bounds and Monte Carlo simulations decreases as the

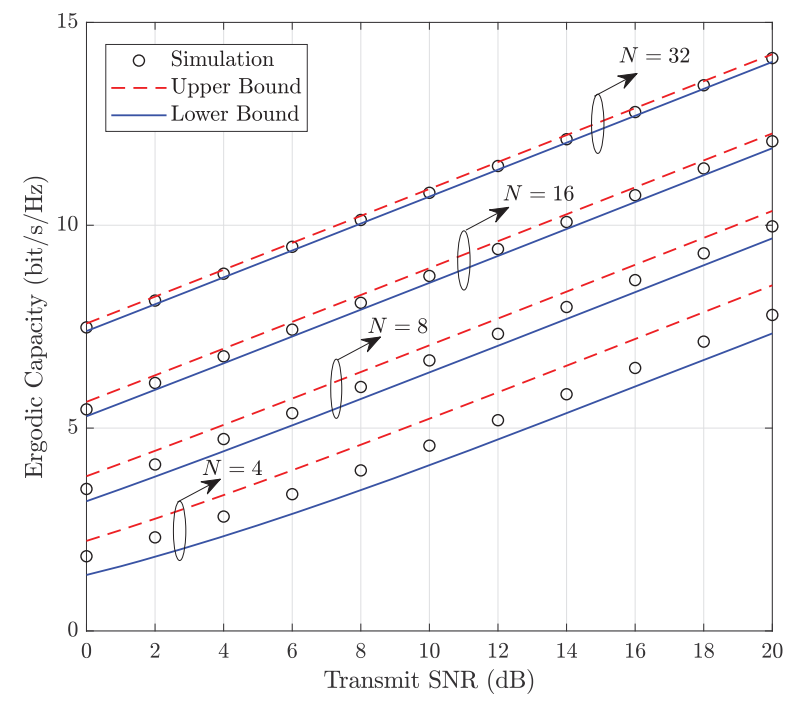

Fig. 4. Upper and lower bounds for the ergodic capacity versus $\gamma_{t}\left(\kappa_{\min }=\right.$ $0.2, \xi=1.5)$.

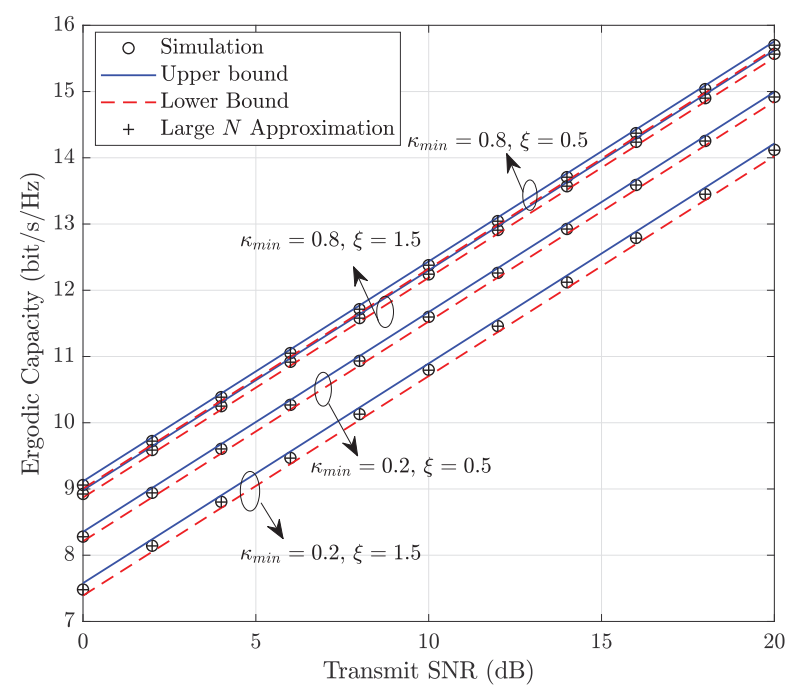

Fig. 5. Comparison of ergodic capacity performances under different $\kappa_{\min }$ and $\xi(N=32)$.

number of reflecting elements $N$ increases, which confirms the tightness of (18) and (19). Additionally, we observe that the EC increases as the SNR $\gamma_{t}$ increases when $N$ is fixed. For example, if $N=32$, setting the SNR to $\gamma_{t}=20 \mathrm{~dB}$ results in $69.5 \%$ of improvement of the upper bound compared to $\gamma_{t}=15 \mathrm{~dB}$. Also, if $\mathrm{SNR}=15 \mathrm{~dB}$, the upper bound of the EC increases by about $18.4 \%$ as $N$ increases from 16 to 32 .

Fig. 5 shows the impact of different system parameters on the EC performance. It is observed that the EC increases by increasing the minimum amplitude $\kappa_{\min }$. In addition, it is observed that the larger the value of $\xi$, the smaller the $\mathrm{EC}$ is. When $\xi$ increases, in fact, the amplitude $\rho_{i}$ decreases for a given phase shift $\phi_{i}$ [31]. Furthermore, we observe that the effect of $\kappa_{\min }$ on the system performance is less significant when $\xi$ increases. We 
also observe that the CLT approximation is accurate even for a moderate number of elements, such as $N=32$.

\section{CONCluSiOn AND Future WORK}

In this paper, we have studied the performance of an RISassisted SISO system under Rayleigh fading channels. Considering a practical phase shift model, we derived exact closed-form expressions for the outage probability and the ergodic capacity. Furthermore, upper and lower bounds for the ergodic capacity were derived, whose accuracy increases with the number of elements of the RIS. Moreover, simplified expressions were obtained under the assumption of a large number of reflecting elements. Our analysis reveals that the system performance improves with the SNR, the number of reflecting elements, the minimum values of the reflection amplitude and a decrease of the steepness of the amplitude function.

This paper can be generalized along several potential research directions. The proposed approach can be generalized to the analysis of multiple-antenna transceivers, to the impact of imperfect CSI, and to discrete phase shifts at the RIS. Furthermore, other performance metrics and their tradeoffs could be analyzed.

\section{APPENDIX A \\ PROOF OF COROLLARY 1}

We first consider the PDF of $M_{i} \triangleq Z_{i} R_{i}$ by using the integral $f_{M_{i}}(m)=\int_{0}^{\infty} f\left(z, \frac{m}{z}\right) \frac{1}{z} d z$ which is given by

$$
\begin{aligned}
f_{M_{i}}(m)= & \frac{2}{\pi \xi\left(1-\kappa_{\min }\right)} \int_{m}^{\frac{m}{\kappa_{\min }}} \frac{1}{z} G_{0,2}^{2,0}\left[z^{2} \mid \frac{1}{\frac{1}{2}, \frac{1}{2}}\right] \\
& \times \frac{\left(\frac{\frac{m}{z}-\kappa_{\min }}{1-\kappa_{\min }}\right)^{1 / \xi-1}}{\sqrt{\left(\frac{\frac{m}{z}-\kappa_{\min }}{1-\kappa_{\min }}\right)^{1 / \xi}-\left(\frac{\frac{m}{z}-\kappa_{\min }}{1-\kappa_{\min }}\right)^{2 / \xi}}} d z .
\end{aligned}
$$

Substituting $\left(\frac{\frac{m}{z}-\kappa_{\min }}{1-\kappa_{\min }}\right)^{1 / \xi}$ with $u$ and applying [34, Eq. (9.31.5)] with some algebraic manipulations, we obtain

$$
\begin{aligned}
f_{M_{i}}(m)= & \frac{2}{\pi m} \int_{0}^{1} \frac{1}{\sqrt{u} \sqrt{1-u}} \\
G_{2,0}^{0,2} & {\left[\frac{\left(\left(1-\kappa_{\min }\right) u^{\xi}+\kappa_{\min }\right)^{2}}{m^{2}} \mid \begin{array}{l}
0,0 \\
-
\end{array}\right] d u . }
\end{aligned}
$$

Using the definition of the Meijer's $G$-function [34, Eq. (9.301)], we write (A.2) as

$$
\begin{aligned}
f_{M_{i}}(m)= & \frac{1}{2 \pi i} \int_{\mathcal{L}_{1}} \Gamma\left(1+s_{1}\right) \Gamma\left(1+s_{1}\right)\left(\frac{\kappa_{\min }}{m}\right)^{2 s_{1}} d s \\
& \times \underbrace{\int_{0}^{1} \frac{1}{\sqrt{u} \sqrt{1-u}}\left[1+a u^{\xi}\right]^{2 s_{1}} d u}_{I_{1}},
\end{aligned}
$$

where $a \triangleq \frac{1-\kappa_{\min }}{\kappa_{\min }}$ and $\mathcal{L}_{i}$ denotes the path of the integration. By employing the integration by parts method, $I_{1}$ can be expressed as

$$
I_{1}=2 \int_{0}^{1} \frac{1}{\sqrt{1-u}}\left(1+a u^{\xi}\right)^{2 s_{1}} d \sqrt{u} .
$$

Substituting $\sqrt{u}$ with $t$, we arrive at the following result

$$
I_{1}=2 \int_{0}^{1}\left(1-t^{2}\right)^{-\frac{1}{2}}\left(1+a t^{2 \xi}\right)^{2 s_{1}} d t \text {. }
$$

Utilizing the Mellin transform of $\left(1-t^{2}\right)^{-\frac{1}{2}}$ [43], we obtain

$$
I_{1}=\frac{1}{2 \pi i} \int_{\mathcal{L}_{2}} \frac{\Gamma\left(\frac{1}{2}\right) \Gamma\left(\frac{s_{2}}{2}\right)}{\Gamma\left(\frac{1+s_{2}}{2}\right)} d s_{2} \int_{0}^{1} t^{-s_{2}}\left(1+a t^{2 \xi}\right)^{2 s_{1}} d t .
$$

With the help of [43], $\left(1+a t^{2 \xi}\right)^{2 s_{1}}$ can be expressed as

$$
\left(1+a t^{2 \xi}\right)^{2 s_{1}}=\frac{1}{2 \pi i} \int_{\mathcal{L}_{3}} \frac{a^{-\frac{s_{3}}{2 \xi}} \Gamma\left(\frac{s_{3}}{2 \xi}\right) \Gamma\left(-2 s_{1}-\frac{s_{3}}{2 \xi}\right)}{2 \xi} t^{-s_{3}} d s_{3},
$$

where $0<\operatorname{Re}\left(s_{3}\right)<-4 \xi \operatorname{Re}\left(s_{1}\right)$. Plugging (A.7) and (A.6) into (A.3), we obtain

$$
\begin{aligned}
& f_{M_{i}}(m)=\frac{1}{\sqrt{\pi} \xi m} H_{2,1: 3,0: 1,1: 1,0}^{0,2: 0: 1: 0} \\
& {\left[\begin{array}{l|l}
\left(1 ;-2,0,-\frac{1}{2 \xi}\right)(0 ; 0,-1,-1) & \\
(-1 ; 0,-1,-1) & \\
(0,1)(0,1)(0,2) & \left(\frac{\kappa_{\min }}{m}\right)^{2}, 1, a^{-\frac{1}{2 \xi}} \\
\left(0, \frac{1}{2}\right) & \\
\left(\frac{1}{2}, \frac{1}{2}\right) & \\
\left(0, \frac{1}{2 \xi}\right) &
\end{array}\right] .}
\end{aligned}
$$

Based on [44], we compute the moment generating function (MGF) of $M_{i}$ defined as $\mathbb{E}\left(e^{-s m}\right)$ with the help of [34, Eq. (3.326.2)]. Since the RVs $M_{i}$ are independent, we can obtain the MGF of the sum $A \triangleq \sum_{i=1}^{N} M_{i}$ as $\tilde{f}_{A}(s)=\prod_{i=1}^{N} \tilde{f}_{M_{i}}(s)$. Then the $\mathrm{CDF}_{F_{A}}(x)$ can be obtained using the inverse Laplace transform of $\tilde{f}_{A}(s) / s$. From (4) with some algebraic manipulations, we arrive at

$$
\begin{aligned}
F_{\gamma}(\gamma)= & \left(\frac{1}{\sqrt{\pi} \xi}\right)^{N}\left(\frac{1}{2 \pi i}\right)^{3 N} \int_{\mathcal{L}_{1}} \ldots \int_{\mathcal{L}_{3 N}} \prod_{n=1}^{N} \\
& \Upsilon\left(s_{3 n-2}, s_{3 n-1}, s_{3 n}\right) \\
& \times \frac{1}{\Gamma\left(1-2 \sum_{m=1}^{N} s_{3 m-2}\right)} \\
& \left(\frac{\sqrt{\overline{\gamma_{t}}} \kappa_{\min }}{\sqrt{\gamma}}\right)^{2} \sum_{m=1}^{N} s_{3 m-2} a^{-\frac{1}{2 \xi} \sum_{m=1}^{N} s_{3 m}} d s_{1} \ldots d s_{3 N},
\end{aligned}
$$

where we define $\Upsilon\left(s_{3 n-2}, s_{3 n-1}, s_{3 n}\right) \triangleq$ $\frac{\Gamma^{2}\left(1+s_{3 n-2}\right) \Gamma\left(\frac{s_{3 n-1}}{2}\right) \Gamma\left(\frac{s_{3 n}}{2 \xi}\right) \Gamma\left(-2 s_{3 n-2}-\frac{s_{3 n}}{2 \xi}\right) \Gamma\left(1-s_{3 n-1}-s_{3 n}\right)}{\Gamma\left(\frac{1+s_{3 n-1}}{2}\right) \Gamma\left(2-s_{3 n-1}-s_{3 n}\right)}$. Finally, 
the proof is completed using the definition of multivariable Fox's $H$-function.

\section{APPENDIX B}

PROOF OF COROLLARY 2

We can derive the mean of the RV $R_{i}$ by using $\mathbb{E}\left(R_{i}\right)=$ $\int_{0}^{\infty} r f_{R_{i}}(r) d r$ and (7), which yields

$$
\begin{aligned}
& \mathbb{E}\left(R_{i}\right) \\
& =\frac{1}{\pi \xi\left(1-\kappa_{\min }\right)} \int_{\kappa_{\min }}^{1} r \frac{\left(\frac{r-\kappa_{\min }}{1-\kappa_{\min }}\right)^{1 / \xi-1}}{\sqrt{\left(\frac{r-\kappa_{\min }}{1-\kappa_{\min }}\right)^{1 / \xi}-\left(\frac{r-\kappa_{\min }}{1-\kappa_{\min }}\right)^{2 / \xi}}} d r .
\end{aligned}
$$

Substituting $\left(\frac{s-\kappa_{\min }}{1-\kappa_{\min }}\right)^{1 / \xi}$ with $t$, we can rewrite (B.1) as

$$
\begin{aligned}
& \mathbb{E}\left(R_{i}\right)=\frac{1}{\pi} \int_{0}^{1}\left(\left(1-\kappa_{\min }\right) t^{\xi}+\kappa_{\min }\right) \frac{1}{\sqrt{t-t^{2}}} d t \\
& =\frac{1-\kappa_{\min }}{\pi} \int_{0}^{1} t^{\xi-\frac{1}{2}}(1-t)^{-\frac{1}{2}} d t+\frac{\kappa_{\min }}{\pi} \int_{0}^{1} t^{-\frac{1}{2}}(1-t)^{-\frac{1}{2}} d t .
\end{aligned}
$$

Then we can obtain (10) with the aid of [34, Eq. (3.191.3)]. In order to obtain the variance of $R_{i}$, we first derive $\mathbb{E}\left(R_{i}^{2}\right)$ following the same approach as for the derivation of (10)

$$
\begin{aligned}
\mathbb{E}\left(R_{i}^{2}\right)= & \frac{\left(1-\kappa_{\min }\right)^{2}}{\pi} B\left(2 \xi+\frac{1}{2}, \frac{1}{2}\right) \\
& +\kappa_{\min }^{2}+\frac{2 \kappa_{\min }\left(1-\kappa_{\min }\right)}{\pi} B\left(\xi+\frac{1}{2}, \frac{1}{2}\right)
\end{aligned}
$$

Using $\operatorname{Var}\left(R_{i}\right)=\mathbb{E}\left(R_{i}^{2}\right)-\left(\mathbb{E}\left(R_{i}\right)\right)^{2}$, we obtain (11) to complete the proof.

\section{APPENDIX C \\ PROOF OF COROLLARY 3}

The PDF of $\gamma$ can be obtained by taking the derivative of (8) and then inserting it into (15). Thus, we obtain the ergodic capacity as

$$
\begin{aligned}
C & =\frac{1}{2}\left(\frac{1}{\sqrt{\pi} \xi}\right)^{N}\left(\frac{1}{2 \pi i}\right)^{3 N} \\
& \times \int_{\mathcal{L}_{1}} \ldots \int_{\mathcal{L}_{3 N}} \prod_{n=1}^{N} \Upsilon\left(s_{3 n-2}, s_{3 n-1}, s_{3 n}\right)\left(\sqrt{\bar{\gamma}_{t}} \kappa_{\min }\right)^{2 \sum_{m=1}^{N} s_{3 m-2}} \\
& \times \frac{a^{-\frac{1}{2 \xi} \sum_{m=1}^{N} s_{3 m}}}{\Gamma\left(-2 \sum_{m=1}^{N} s_{3 m-2}\right)} d s_{1} \ldots d s_{3 N} \\
& \underbrace{\int_{0}^{\infty} \log _{2}(1+\gamma)(\gamma)^{-\sum_{m=1}^{N} s_{3 m-2}-1} d \gamma}_{I_{2}} .
\end{aligned}
$$

Employing [35, Eq. (07.34.03.0456.01)], we can express $I_{2}$ as

$$
I_{2}=\frac{1}{\ln 2} \int_{0}^{\infty} G_{2,2}^{1,2}\left[\gamma \mid \begin{array}{l}
-\sum_{m=1}^{N} s_{3 m-2},-\sum_{m=1}^{N} s_{3 m-2} \\
-\sum_{m=1}^{N} s_{3 m-2},-\sum_{m=1}^{N} s_{3 m-2}-1
\end{array}\right] d \gamma
$$

With the help of [34, Eq. (9.301)] and the Laplace transform of the Meijer's $G$-function [35, Eq. (07.34.22.0003.01)], we obtain (C.5) as shown at the bottom of this page. By defining $\mathcal{L}(p(z))=P(s)$ and utilizing the final value theorem $\quad \lim _{t \rightarrow \infty}\left(\int_{0}^{t} p(z) d z\right)=\lim _{s \rightarrow 0^{+}} s \mathcal{L}\left(\int_{0}^{t} p(z) d z\right)=$ $\lim _{s \rightarrow 0^{+}} s \frac{P(s)}{s}=\lim _{s \rightarrow 0^{+}} P(s)$, we can rewrite the ergodic capacity as (C.6), which is given at the top of the next page, where $s$ is a number close to zero.

Using the definition of the multivariable Fox's $H$-function, we obtain (16) to conclude the proof.

\section{APPENDIX D}

\section{PROOF OF COROLLARY 4}

By using (4) and (17), we can rewrite (15) as

$$
C \leq C^{\mathrm{ub}}=\log _{2}[1+\underbrace{\mathbb{E}\left(\left(\sum_{i=1}^{N} \alpha_{i} \beta_{i} R_{i}\right)^{2} \gamma_{t}\right)}_{I_{3}}] .
$$

$$
\begin{aligned}
& \mathcal{L}\left\{G_{2,2}^{1,2}\left[\gamma \mid \begin{array}{l}
-\sum_{m=1}^{N} s_{3 m-2},-\sum_{m=1}^{N} s_{3 m-2} \\
-\sum_{m=1}^{N} s_{3 m-2},-\sum_{m=1}^{N} s_{3 m-2}-1
\end{array}\right]\right\}=\frac{1}{s} G_{3,2}^{1,3}\left[\frac{1}{s} \mid \begin{array}{l}
0,-\sum_{m=1}^{N} s_{3 m-2},-\sum_{m=1}^{N} s_{3 m-2} \\
-\sum_{m=1}^{N} s_{3 m-2},-\sum_{m=1}^{N} s_{3 m-2}-1
\end{array}\right] \\
& =\frac{1}{s} \int_{\mathcal{L}_{3 N+1^{*}}} \frac{\Gamma\left(-\sum_{m=1}^{N} s_{3 m-2}+s_{3 N+1}\right) \Gamma\left(1-s_{3 N+1}\right) \Gamma^{2}\left(1+\sum_{m=1}^{N} s_{3 m-2}-s_{3 N+1}\right)}{\Gamma\left(2+\sum_{m=1}^{N} s_{3 m-2}-s_{3 N+1}\right)} s^{s_{3 N+1}} d s_{3 N+1}
\end{aligned}
$$




$$
\begin{aligned}
C= & \frac{1}{2 s \ln 2}\left(\frac{1}{\sqrt{\pi} \xi}\right)^{N}\left(\frac{1}{2 \pi i}\right)^{3 N+1} \int_{\mathcal{L}_{1}} \ldots \int_{\mathcal{L}_{3 N+1}} \prod_{n=1}^{N} \Upsilon\left(s_{3 n-2}, s_{3 n-1}, s_{3 n}\right) \\
& \times \frac{\Gamma\left(-\sum_{m=1}^{N} s_{3 m-2}+s_{3 N+1}\right) \Gamma\left(1-s_{3 N+1}\right) \Gamma^{2}\left(1+\sum_{m=1}^{N} s_{3 m-2}-s_{3 N+1}\right)}{\Gamma\left(-2 \sum_{m=1}^{N} s_{3 m-2}\right) \Gamma\left(2+\sum_{m=1}^{N} s_{3 m-2}-s_{3 N+1}\right)} \\
& \times\left(\sqrt{\bar{\gamma}} \kappa_{\text {min }}\right)^{2} \sum_{m=1}^{N} s_{3 m-2} a^{-\frac{1}{2 \xi} \sum_{m=1}^{N} s_{3 m}} s^{s_{3 N+1}} d s_{1} \ldots d s_{3 N+1}
\end{aligned}
$$

The integral $I_{3}$ can be decomposed as

$$
I_{3}=\gamma_{t} \mathbb{E}\left[\sum_{i=1}^{N}\left(\alpha_{i} \beta_{i} R_{i}\right)^{2}+2 \sum_{i=1}^{N-1} \sum_{k=i+1}^{N} \alpha_{i} \beta_{i} R_{i} \alpha_{k} \beta_{k} R_{k}\right]
$$

Applying (10) and (11) into (D.2), we obtain

$$
\begin{array}{r}
\mathbb{E}\left[\sum_{i=1}^{N}\left(\alpha_{i} \beta_{i} R_{i}\right)^{2}\right]=N\left(\delta+\varepsilon^{2}\right), \\
\mathbb{E}\left[\sum_{i=1}^{N-1} \sum_{k=i+1}^{N} \alpha_{i} \beta_{i} R_{i} \alpha_{k} \beta_{k} R_{k}\right]=\frac{N(N-1) \pi^{2} \varepsilon^{2}}{32} .
\end{array}
$$

Thus, by substituting (D.2), (D.3) and (D.4) into (D.1), we obtain $\mathbb{E}(\gamma)$ and the upper bound for the ergodic capacity is derived as in (18).

Next, according to [45], we apply the Taylor series expansion of $1 / \gamma$ around $\mathbb{E}(\gamma)$ and obtain

$$
\mathbb{E}(1 / \gamma) \approx 1 / \mathbb{E}(\gamma)+\operatorname{Var}(\gamma) /[\mathbb{E}(\gamma)]^{3}
$$

The variance $\operatorname{Var}(\gamma)$ can be obtained as follows. From (4), we can write

$\operatorname{Var}(\gamma)=\operatorname{Var}\left(\sum_{i=1}^{N}\left(\alpha_{i} \beta_{i} R_{i}\right)^{2}+2 \sum_{i=1}^{N-1} \sum_{k=i+1}^{N} \alpha_{i} \beta_{i} R_{i} \alpha_{k} \beta_{k} R_{k}\right)$

Using $\operatorname{Var}\left(\sum_{i=1}^{N} X_{i}\right)=\sum_{i=1}^{N} \operatorname{Var}\left(X_{i}\right)+2 \sum_{i=1}^{N-1} \sum_{k=i+1}^{N}$ $\operatorname{Cov}\left(X_{i}, X_{k}\right), \quad \operatorname{Var}\left(X_{i}\right)=\mathbb{E}\left(X_{i}^{2}\right)-\mathbb{E}\left(X_{i}\right) \mathbb{E}\left(X_{i}\right)$, $\operatorname{Cov}\left(X_{i}, X_{k}\right)=\mathbb{E}\left(X_{i} X_{k}\right)-\mathbb{E}\left(X_{i}\right) \mathbb{E}\left(X_{k}\right)$ and $\mathbb{E}\left(x^{n}\right)=$ $\int_{0}^{\infty} x^{n} f_{X}(x) d x$, the variance of $\gamma$ can be expressed as in (20), which completes the proof.

\section{APPENDIX E \\ PROOF OF COROLLARY 5}

The ergodic capacity can be written as

$$
C=\frac{B}{\ln 2} \int_{0}^{\infty} \ln (1+\gamma) f_{\gamma}(\gamma) d \gamma
$$

Substituting (13) into (E.1), we obtain

$$
\begin{aligned}
C & =\frac{B}{2 \eta^{2} \gamma_{t} \ln 2}\left(\frac{1}{\gamma_{t} \lambda}\right)^{-\frac{1}{4}} \exp \left(-\frac{\lambda}{2 \eta^{2}}\right) \\
& \times \int_{0}^{\infty} \ln (1+\gamma) \exp \left(-\frac{\gamma}{2 \gamma_{t} \eta^{2}}\right) I_{-\frac{1}{2}}\left(\frac{\sqrt{\lambda}}{\sqrt{\gamma_{t}} \eta^{2}} \sqrt{\gamma}\right) \gamma^{-\frac{1}{4}} d \gamma
\end{aligned}
$$

With the aid of [35, Eq. (07.34.26.0008.01)] and [34, Eq. (9.31.5)], we can rewrite $I_{-\frac{1}{2}}\left(\frac{\sqrt{\gamma \lambda}}{\sqrt{\gamma_{t}} \eta^{2}}\right)$ in terms of the Fox's $H$ function as

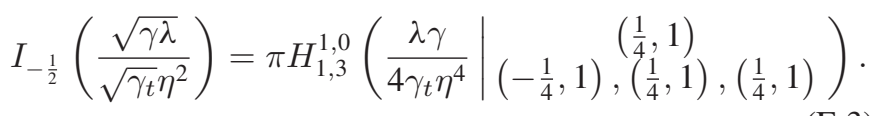

Furthermore, by employing [35, Eq. (07.34.03.0228.01)], [35, Eq. (07.34.03.0456.01)] and [35, Eq. (07.34.26.0008.01)], the logarithm function and the exponential function can be written in terms of the Fox's $H$-function as

$$
\begin{aligned}
\ln (1+\gamma) & =H_{2,2}^{1,2}\left(\gamma \mid \begin{array}{l}
(1,1),(1,1) \\
(1,1),(0,1)
\end{array}\right), \\
\exp \left(-\frac{\gamma}{2 \gamma_{t} \eta^{2}}\right) & =H_{0,1}^{1,0}\left(\frac{\gamma}{2 \gamma_{t} \eta^{2}} \mid \begin{array}{c}
- \\
(0,1)
\end{array}\right) .
\end{aligned}
$$

Substituting (E.3), (E.4) and (E.5) into (E.2) and utilizing the definition of the multivariable Fox's $H$-function, the proof is completed and (21) is proved.

\section{REFERENCES}

[1] J. Zhang, E. Björnson, M. Matthaiou, D. W. K. Ng, H. Yang, and D. J. Love, "Prospective multiple antenna technologies for beyond 5G," IEEE J. Sel. Areas Commun., vol. 38, no. 8, pp. 1637-1660, Aug. 2020.

[2] Q. Wu and R. Zhang, "Beamforming optimization for wireless network aided by intelligent reflecting surface with discrete phase shifts," IEEE Trans. Commun., vol. 68, no. 3, pp. 1838-1851, Mar. 2020.

[3] M. Di Renzo et al., "Smart radio environments empowered by reconfigurable AI meta-surfaces: An idea whose time has come," EURASIP J. Wireless Commun. Netw., vol. 2019, no. 1, p. 129, May 2019.

[4] M. Di Renzo et al., "Smart radio environments empowered by reconfigurable intelligent surfaces: How it works, state of research, and the road ahead," IEEE J. Sel. Areas Commun., vol. 38, no. 11, pp. 2450-2525, Nov. 2020

[5] M. Di Renzo et al., "Reconfigurable intelligent surfaces vs. relaying: Differences, similarities, and performance comparison," IEEE Open J. Commun. Soc., vol. 1, pp. 798-807, Jun. 2020.

[6] E. Basar, M. Di Renzo, J. De Rosny, M. Debbah, M.-S. Alouini, and R. Zhang, "Wireless communications through reconfigurable intelligent surfaces," IEEE Access, vol. 7, pp. 116 753-116 773, Aug. 2019. 
[7] C. Huang et al., "Holographic MIMO surfaces for $6 \mathrm{G}$ wireless networks: Opportunities, challenges, and trends," IEEE Wireless Commun., vol. 27, no. 5, pp. 118-125, Oct. 2020.

[8] Y. Liu et al., "Reconfigurable intelligent surfaces: Principles and opportunities," [Online]. Available: https://arxiv.org/abs/2007.03435

[9] W. Tang et al., "Wireless communications with reconfigurable intelligent surface: Path loss modeling and experimental measurement," IEEE Trans. Wireless Commun., vol. 20, no. 1, pp. 421-439, Jan. 2021.

[10] H. Du, J. Zhang, J. Cheng, and B. Ai, "Millimeter wave communications with reconfigurable intelligent surfaces: Performance analysis and optimization," IEEE Trans. Commun., vol. 69, no. 4, pp. 2752-2768, Apr. 2021.

[11] Y. Chen, Y. Wang, J. Zhang, and Z. Li, "Resource allocation for intelligent reflecting surface aided vehicular communications," IEEE Trans. Veh. Technol., vol. 69, no. 10, pp. 12 321-12 326, Oct. 2020.

[12] L. Yang, F. Meng, J. Zhang, M. O. Hasna, and M. Di Renzo, "On the performance of RIS-assisted dual-hop UAV communication systems," IEEE Trans. Veh. Technol., vol. 69, no. 9, pp. 10 385-10 390, Sep. 2020.

[13] L. Yang, Y. Yang, M. O. Hasna, and M.-S. Alouini, "Coverage, probability of SNR gain, and DOR analysis of RIS-aided communication systems,' IEEE Wireless Commun. Lett., vol. 9, no. 8, pp. 1268-1272, Aug. 2020.

[14] R. C. Ferreira, M. S. P. Facina, F. A. P. De Figueiredo, G. Fraidenraich, and E. R. De Lima, "Bit error probability for large intelligent surfaces under double-nakagami fading channels," IEEE Open J. Commun. Soc., vol. 1, pp. 750-759, May 2020.

[15] D. Li, "Ergodic capacity of intelligent reflecting surface-assisted communication systems with phase errors," IEEE Commun. Lett., vol. 24, no. 8 , pp. 1646-1650, Aug. 2020.

[16] A.-A. A. Boulogeorgos and A. Alexiou, "Ergodic capacity analysis of reconfigurable intelligent surface assisted wireless systems," in Proc. IEEE 3rd 5 G World Forum, 2020, pp. 395-400.

[17] C. Guo, Y. Cui, F. Yang, and L. Ding, "Outage probability analysis and minimization in intelligent reflecting surface-assisted MISO systems," IEEE Commun. Lett., vol. 24, no. 7, pp. 1563-1567, Jul. 2020.

[18] S. Atapattu, R. Fan, P. Dharmawansa, G. Wang, J. Evans, and T. A. Tsiftsis, "Reconfigurable intelligent surface assisted two-way communications: Performance analysis and optimization," IEEE Trans. Commun., vol. 68, no. 10 , pp. 6552-6567, Oct. 2020.

[19] I. Trigui, W. Ajib, and W. Zhu, "A comprehensive study of reconfigurable intelligent surfaces in generalized fading," [Online]. Available: https:// arxiv.org/abs/2004.02922

[20] M.-A. Badiu and J. P. Coon, "Communication through a large reflecting surface with phase errors," IEEE Wireless Commun. Lett., vol. 9, no. 2, pp. 184-188, Feb. 2020.

[21] H. Zhang, B. Di, L. Song, and Z. Han, "Reconfigurable intelligent surfaces assisted communications with limited phase shifts: How many phase shifts are enough?," IEEE Trans. Veh. Technol., vol. 69, no. 4, pp. 4498-4502. Apr. 2020.

[22] S. Zhou, W. Xu, K. Wang, M. Di Renzo, and M.-S. Alouini, "Spectral and energy efficiency of IRS-assisted MISO communication with hardware impairments," IEEE Wireless Commun. Lett., vol. 9, no. 9, pp. 1366-1369, Apr. 2020.

[23] P. Xu, G. Chen, Z. Yang, and M. Di Renzo, "Reconfigurable intelligent surfaces-assisted communications with discrete phase shifts: How many quantization levels are required to achieve full diversity?," IEEE Wireless Commun. Lett., vol. 10, no. 2, pp. 358-362, Feb. 2021.

[24] P. Xu, G. Chen, G. Pan, and M. Di Renzo, "Ergodic secrecy capacity of RIS-assisted communication systems in the presence of discrete phase shifts and multiple eavesdroppers," [Online]. Available: https://arxiv.org/ abs/2009.00517

[25] L. Yang, J. Yang, W. Xie, M. O. Hasna, T. Tsiftsis, and M. Di Renzo, "Secrecy performance analysis of RIS-aided wireless communication systems," IEEE Trans. Veh. Technol., vol. 69, no. 10, pp. 12296-12300, Oct. 2020.

[26] L. Yang, F. Meng, Q. Wu, D. Costa, and M.-S. Alouini, "Accurate closed-form approximations to channel distributions of RIS-aided wireless systems," IEEE Wireless Commun. Lett., vol. 9, no. 11, pp. 1985-1989, Nov. 2020.

[27] X. Qian, M. Di Renzo, J. Liu, A. Kammoun, and M.-S. Alouini, "Beamforming through reconfigurable intelligent surfaces in single-user MIMO systems: SNR distribution and scaling laws in the presence of channel fading and phase noise," IEEE Wireless Commun. Lett., vol. 10, no. 1, pp. 77-81, Jan. 2021
[28] C. Huang, A. Zappone, G. C. Alexandropoulos, M. Debbah, and C. Yuen, "Reconfigurable intelligent surfaces for energy efficiency in wireless communication," IEEE Trans. Wireless Commun., vol. 18, no. 8, pp. 4157-4170, Aug. 2019.

[29] C. Huang et al., "Multi-hop RIS-empowered terahertz communications: A DRL-based hybrid beamforming design," in Proc. IEEE Globecom Workshops, Dec. 2020, pp. 1-6.

[30] I. Trigui, W. Ajib, W.-P. Zhu, and M. Di Renzo, "Performance evaluation and diversity analysis of RIS-assisted communications over generalized fading channels in the presence of phase noise," [Online]. Available: https: //arxiv.org/abs/2011.12260

[31] S. Abeywickrama, R. Zhang, Q. Wu, and C. Yuen, "Intelligent reflecting surface: Practical phase shift model and beamforming optimization," IEEE Trans. Commun., vol. 68, no. 9, pp. 5849-5863, Sep. 2020.

[32] G. Gradoni and M. Di Renzo, "End-to-end mutual-coupling-aware communication model for reconfigurable intelligent surfaces: An electromagnetic-compliant approach based on mutual impedances," IEEE Wireless Commun. Lett., vol. 10, no. 5, pp. 938-942, May 2021.

[33] I. Z. Kovacs, P. C. F. Eggers, K. Olesen, and L. G. Petersen, "Investigations of outdoor-to-indoor mobile-to-mobile radio communication channels," Proc. IEEE 56th Veh. Technol. Conf., vol. 1, Sep. 2002, pp. 430-434.

[34] I. S. Gradshteyn and I. M. Ryzhik, Table of Integrals, Series and Products, 7th ed. Burlington, MA, USA: Academic, 2007.

[35] Wolfram, "The Wolfram functions site," [Online]. Available: http:// functions.wolfram.com

[36] J. G. Proakis and M. Salehi, Digital Communications, 4th ed. New York, NY, USA: McGraw Hill, 2001.

[37] A. M. Mathai, R. K. Saxena, and H. J. Haubold, The H-Function: Theory and Applications. New York, NY, USA: Springer-Verlag, 2010.

[38] H. Lei, I. S. Ansari, G. Pan, B. Alomair, and M.-S. Alouini, "Secrecy capacity analysis over $\alpha-\mu$ fading channels," IEEE Commun. Lett., vol. 21, no. 6, pp. 1445-1448, Jun. 2017.

[39] J. Zhang, H. Du, P. Zhang, J. Cheng, and L. Yang, "Performance analysis of 5G mobile relay systems for high-speed trains," IEEE J. Sel. Areas Commun., vol. 38, no. 12, pp. 2760-2772, Dec. 2020.

[40] H. Chergui, M. Benjillali, and M.-S. Alouini, "Rician $K$-factor-based analysis of XLOS service probability in 5G outdoor ultra-dense networks," IEEE Wireless Commun. Lett., vol. 8, no. 2, pp. 428-431, Apr. 2019.

[41] H. R. Alhennawi, M. M. H. El Ayadi, M. H. Ismail, and H. A. M. Mourad, "Closed-form exact and asymptotic expressions for the symbol error rate and capacity of the $H$-function fading channel," IEEE Trans. Veh. Technol., vol. 65, no. 4, pp. 1957-1974, Apr. 2016.

[42] V. M. Kapinas, S. K. Mihos, and G. K. Karagiannidis, "On the monotonicity of the generalized Marcum and Nuttall $Q$-functions," IEEE Trans. Inf. Theory, vol. 55, no. 8, pp. 3701-3710, Aug. 2009.

[43] H. Bateman, Tables of Integral Transforms, vol. 1. Bateman Manuscript Project: McGraw-Hill, New York, 1954.

[44] Y. Abo Rahama, M. H. Ismail, and M. S. Hassan, "On the sum of independent fox's $H$-function variates with applications," IEEE Trans. Veh. Technol., vol. 67, no. 8, pp. 6752-6760, Aug. 2018.

[45] Q. Zhang, S. Jin, K.-K. Wong, H. Zhu, and M. Matthaiou, "Power scaling of uplink massive MIMO systems with arbitrary-rank channel means," IEEE J. Sel. Top. Signal Process, vol. 8, no. 5, pp. 966-981, Oct. 2014. 